\title{
Some Remarks About the Cyclic Homology of Skew PBW Extensions
}

\author{
Algunas observaciones sobre la homología cíclica de extensiones $P B W$ torcidas
}

Milton Armando Reyes Villamil ${ }^{\mathrm{a} *}$

Héctor Julio Suárez Suárez ${ }^{\mathrm{b}}$

Recepción: 11-feb-2016

Aceptación: 29-may-2016

\begin{abstract}
We study the cyclic homology for a class of noncommutative polynomial rings known as skew $P B W$ extensions. We obtain explicit computations for some important families of such extensions over fields. In particular, we consider the cyclic homology of skew $P B W$ extensions of derivation type, certain classes of Ore extensions, operator algebras, difusion algebras, quantum algebras and 3-dimensional skew polynomial algebras.
\end{abstract}

Key words: Cyclic homology, Filtered rings, Skew $P B W$ extensions.

\section{Resumen}

Estudiamos la homología cíclica de una clase de anillos de polinomios no conmutativos denominados extensiones $P B W$ torcidas. Obtenemos cálculos explícitos para algunas familias importantes de este tipo de extensiones sobre cuerpos. En particular, consideramos la homología cíclica de las extensiones $P B W$ torcidas de tipo derivación, ciertas clases de extensiones de Ore, álgebras de operadores, álgebras de difusión, álgebras cuánticas y álgebras de polinomios torcidos 3-dimensionales.

Palabras clave: Anillos filtrados, Extensiones $P B W$ torcidas, Homología cíclica.

\section{Introduction}

Cyclic homology of algebras was discovered by Connes in the formulation of noncommutative differential geometry [3]. In connection with the pairing with algebraic or topological $K$-theory, cyclic homology is quite useful also for the study of $K$ theory. For instance, Connes uses cyclic cocycles to express certain characteristic classes of a foliation in connection with the topological $K$-theory of the associated foliation $C^{*}$-algebra. In this context, it seems to be important to compute cyclic cohomology of interesting algebras, which appear in differential topology or in algebraic geometry. Cyclic homology has been studied in a series of papers as a noncommutative generalization of de Rham cohomology (cf. [14], [23], [4], [5]) in order to interpret index theorems for non-commutative Banach algebras, via a generalization of the Chern character, where it

\footnotetext{
${ }^{a}$ Seminario de Álgebra Constructiva - SAC ${ }^{2}$, Departamento de Matemáticas, Universidad Nacional de Colombia - sede Bogotá. *Correo electrónico: mareyesv@ unal.edu.co

${ }^{b}$ Escuela de Matemáticas y Estadística, Universidad Pedagógica y Tecnológica de Colombia, Tunja.
} 
was shown in [3] that cyclic homology of $C^{\infty}(M)$ recovers the $\mathbb{C}$-coefficient de Rham homology of the compact smooth manifold $M$. Cyclic homology was also shown to be the primitive part of the Lie algebra homology of matrices by Quillen and Loday [14]. This relationship shows that cyclic homology can be considered as a Lie analogue of algebraic $K$-theory and it is sometimes referred to as non-commutative differential geometry. Following [15], the cyclic homology of an $k$-algebra $B$ ( $k$ being a commutative ring) consists of abelian groups $H C_{n}(B), n \geq 0$. If $k$ is a field with characteristic zero, these groups are the homology groups of the quotient of the Hochschild complex by the action of the finite cyclic groups; this is the reason for the term "cyclic". The notation $H C$ was for "Homologie de Connes", but soon became "Homologie Cyclique".

Since we are interested in computing the cyclic homology groups of skew PBW (PBW denotes Poincaré-Birkhoff-Witt) extensions introduced in [7], in this paper we have compiled some facts about these groups for certain examples of this kind of extensions. We consider that this study enriches the study of non-commutative differential geometry of a considerable number of noncommutative rings and quantum groups (for instance, quantum spaces whose cyclic homologies were known [4], quasicommutative algebras [11], $\mathbb{Q}$-difference operators [9], Ore extensions and some quantum algebras [10]). The techniques used here are fairly standard and follow the same path as other text on the subject. The results presented are new for skew PBW extensions and all they are similar to others existing in the literature. In this way, we continue the task of studying several properties of skew PBW extensions and its relationship with other noncommutative rings (see [1], [8], [12], [13], [17], [18], [19], [20], [22], [24] and others).

The paper is organized as follows. In Section 2 we describe the skew $P B W$ extensions. In Section 3 we recall the higher algebraic $K$-theory of these extensions following [13], and then we present the key results of this paper concerning about cyclic homology of these rings. Finally, in Section 4 we illustrate the results about cyclic homology of Section 3 with several examples such as Ore extensions, diffusion algebras, operator algebras and 3-dimensional skew polynomial algebras.

\section{Skew $P B W$ Extensions}

Definition 1 ([7], Definition 1). Let $R$ and $A$ be rings. We say that $A$ is a skew PBW extension of $R$ (also called a $\sigma-P B W$ extension of $R$ ) if the following conditions hold:

(i) $R \subseteq A$.

(ii) There exist finite elements $x_{1}, \ldots, x_{n} \in A$ such $A$ is a left $R$-free module with basis

$$
\begin{gathered}
\operatorname{Mon}(A):=\left\{x^{\alpha}=x_{1}^{\alpha_{1}} \cdots x_{n}^{\alpha_{n}} \mid \alpha=\right. \\
\left.\left(\alpha_{1}, \ldots, \alpha_{n}\right) \in \mathbb{N}^{n}\right\} .
\end{gathered}
$$

We say also that $A$ is a left polynomial ring over $R$ with respect to the set of variables $\left\{x_{1}, \ldots, x_{n}\right\}$ and $\operatorname{Mon}(A)$ is the set of standard monomials of $A$. In addition, $x_{1}^{0} \cdots x_{n}^{0}:=1 \in \operatorname{Mon}(A)$.

(iii) For every $1 \leq i \leq n$ and $r \in R \backslash\{0\}$ there exists $c_{i, r} \in R \backslash\{0\}$ such that $x_{i} r-c_{i, r} x_{i} \in R$.

(iv) For every $1 \leq i, j \leq n$ there exists $c_{i, j} \in R \backslash\{0\}$ such that $x_{j} x_{i}-c_{i, j} x_{i} x_{j} \in R+R x_{1}+\cdots+R x_{n}$.

Under these conditions we will write $A:=$ $\sigma(R)\left\langle x_{1}, \ldots, x_{n}\right\rangle$.

The following proposition justifies the notation for skew $P B W$ extensions. Before, we recall the notion of skew polynomial ring. If $B$ is a ring and $\sigma$ is a ring endomorphism $\sigma: B \rightarrow B$, a $\sigma$-derivation $\delta$ : $B \rightarrow B$ satisfies by definition $\delta(r+s)=\delta(r)+\delta(s)$, and $\delta(r s)=\sigma(r) \delta(s)+\delta(r) s$ for all $r, s \in B$. If $y$ is an indeterminate, and $y b=\sigma(b) y+\delta(b)$, for any $b \in B$, we denote this noncommutative ring as $B[y ; \sigma, \delta]$ which is called a skew polynomial ring.

Proposition 2 ([7], Proposition 3). Let A be a skew $P B W$ extension of $R$. Then, for every $1 \leq i \leq n$, there exist an injective ring endomorphism $\sigma_{i}: R \rightarrow R$ and a $\sigma_{i}$-derivation $\delta_{i}: R \rightarrow R$ such that $x_{i} r=$ $\sigma_{i}(r) x_{i}+\delta_{i}(r)$ for each $r \in R$.

Definition 3. Let $A$ be a skew $P B W$ extension.

(a) ([7], Definition 4.) A is quasi-commutative if the conditions (iii) and (iv) in Definition 1 are replaced by

(iii') For every $1 \leq i \leq n$ and $r \in R \backslash\{0\}$ there exists $c_{i, r} \in R \backslash\{0\}$ such that $x_{i} r=c_{i, r} x_{i}$.

(iv') For every $1 \leq i, j \leq n$ there exists $c_{i, j} \in$ $R \backslash\{0\}$ such that $x_{j} x_{i}=c_{i, j} x_{i} x_{j}$.

(b) $A$ is bijective if $\sigma_{i}$ is bijective for every $1 \leq i \leq n$ and $c_{i, j}$ is invertible for any $1 \leq i<j \leq n$. 
(c) ([12], Definition 2.3.) A is a skew PBW extension of derivation type if $\sigma_{i}=\mathrm{id}_{R}$ for $1 \leq i \leq n$.

Definition 4. A filtered ring is a ring $B$ with a family $F B=\left\{F_{n} B \mid n \in \mathbb{Z}\right\}$ of additive subgroups of $B$ where we have the ascending chain $\cdots \subset F_{n-1} B \subset$ $F_{n} B \subset \cdots$ such that $1 \in F_{0} B$ and $F_{n} B F_{m} B \subseteq F_{n+m} B$ for all $n, m \in \mathbb{Z}$. From a filtered ring $B$ it is possible to construct its associated graded ring $G(B)$ taking $G(B)_{n}:=F_{n} B / F_{n-1} B$.

The next proposition computes the graduation of a general skew $P B W$ extension of a ring $R$. This result will very important in Proposition 12.

Proposition 5 ([13], Theorem 2.2). Let $A$ be an arbitrary skew $P B W$ extension of $R$. Then, $A$ is a filtered ring with increasing filtration given by

$$
F_{m} A:= \begin{cases}R & \text { if } m=0 \\ \{f \in A \mid \operatorname{deg}(f) \leq m\} & \text { if } m \geq 1\end{cases}
$$

and the corresponding graded ring $G(A)$ is a quasicommutative skew $P B W$ extension of $R$. Moreover, if $A$ is bijective, then $G(A)$ is a quasi-commutative bijective skew $P B W$ extension of $R$.

Remark 6. The associated graded ring $G(A)$ is the skew $P B W$ extension of $R$ generated by the variables $z_{1}, \ldots, z_{n}$ with the relations $z_{i} r=c_{i, r} z_{i}, z_{j} z_{i}=c_{i, j} z_{i} z_{j}$, for $1 \leq i \leq n$, where $c_{i, r}, c_{i, j}$ are the same constants that define $A$. See [13], Proposition 2.1 for a proof of this assertion.

Proposition 7 establishes the relation between skew $P B W$ extensions and iterated skew polynomial rings in the sense of Proposition 2.

Proposition 7 ([13], Theorem 2.3). Let A be a quasicommutative skew PBW extension of a ring $R$. Then (i) A is isomorphic to an iterated skew polynomial ring, and (ii) if $A$ is bijective, each endomorphism of the skew polynomial ring in (i) is an isomorphism.

\section{Algebraic $K$-Theory and Lie Analogue of Higher Algebraic $K$-Theory}

As we said in the Introduction, cyclic homology was shown to be the primitive part of the Lie algebra homology of matrices by Quillen and Loday [14]. This relationship shows that cyclic homology can be considered as a Lie analogue of algebraic $K$-theory and it is sometimes referred to as non-commutative differential geometry. With this in mind, in the first part of this section we recall the higher algebraic $K$-theory of skew $P B W$ extensions following [13], while the second part treats with the cyclic homology of skew $P B W$ extensions.

\subsection{Higher Algebraic $K$-Theory of Skew $P B W$ Extensions}

Quillen [16] proposed defining the higher algebraic $K$-theory of a ring $B$ to be the homotopy groups of a certain topological space which he called " $B G L(R)^{+}$". In fact, he provided two fundamentally different ways of defining higher algebraic $K$-groups, one homotopy theoretic and the other category theoretic. Following a categoric treatment, Lezama and Reyes [13] computed the higher algebraic $K$-theory of bijective skew $P B W$ extensions.

Proposition 8 ([13], Theorem 5.1). Let $R$ be a left Noetherian left regular ring. If $A$ is a bijective skew $P B W$ extension of $R$, then $K_{i}(A) \cong K_{i}(R)$ for all $i \geq 0$.

With this result, it is possible to obtain the Quillen's groups $K_{i}, i \geq 0$ for several families of noncommutative rings which are examples of bijective skew $P B W$ extensions. For instance, $P B W$ extensions, Ore extensions of bijective type, operator algebras, diffusion algebras, some quantum algebras, and 3-dimensional skew polynomial algebras, and some localizations of skew $P B W$ extensions (see [13] for a detailed reference of every family). A detailed list of these groups can be found it in [13].

\subsection{Lie Analogue of Higher Algebraic $K$-Theory of Skew $P B W$ Extensions}

We recall the definitions of Hochschild and cyclic homology following [6]. For more details see [4], [5], [14], [15] or [23].

Definition 9. Let $B$ be an associative algebra over a commutative unital ring $k, k \subset B$. The Hochschild homology of $B$, denoted by $H_{*}(B)$, is defined to be the homology of the following complex

$$
0 \leftarrow C_{0}(B) \stackrel{d}{\leftarrow} C_{1}(B) \stackrel{d}{\leftarrow} C_{2}(B) \stackrel{d}{\leftarrow} \cdots,
$$

where $C_{n}(B)=B^{\otimes_{n+1}}$, and $d: C_{n}(B) \rightarrow C_{n-1}(B)$ such that 


$$
\begin{aligned}
& d\left(b_{0} \otimes b_{1} \otimes \cdots \otimes b_{n}\right) \\
& =\sum_{i=0}^{n-1}(-1)^{i} b_{0} \otimes b_{1} \otimes \cdots \otimes b_{i} b_{i+1} \otimes \cdots \otimes b_{n} \\
& \quad+(-1)^{n} b_{n} b_{0} \otimes b_{1} \otimes \cdots \otimes b_{n-1} . \quad
\end{aligned}
$$

The cyclic homology of $B$, denoted by $H C_{*}(B)$, is defined to be the total homology of the following double complex

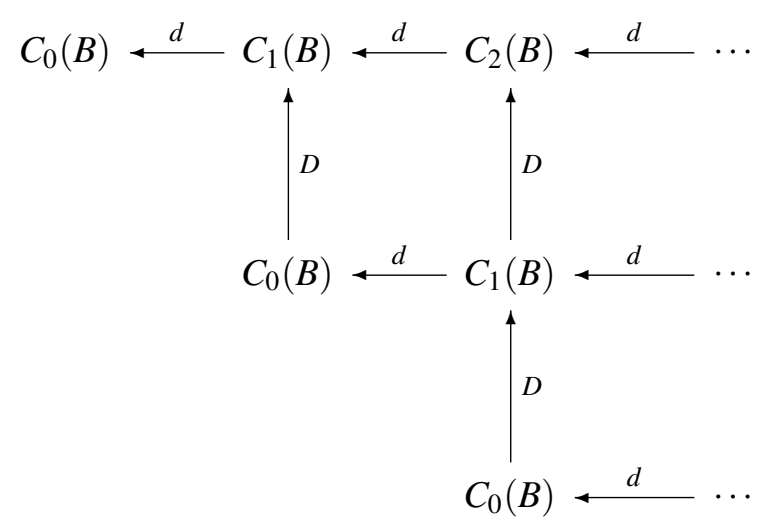

where $D: C_{n}(B) \rightarrow C_{n+1}(B)$ such that

$$
\begin{aligned}
& D\left(b_{0} \otimes b_{1} \otimes \cdots \otimes b_{n}\right) \\
& =\sum_{i=0}^{n}(-1)^{n i}\left(1 \otimes b_{i} \otimes \cdots \otimes b_{n} \otimes b_{0} \otimes \cdots \otimes b_{i-1}\right. \\
& \left.+(-1)^{n} b_{i} \otimes \cdots \otimes b_{n} \otimes b_{0} \otimes \cdots \otimes b_{i-1} \otimes 1\right) .
\end{aligned}
$$

In [2] Hochschild homology and cyclic homology are denoted by $H_{i}(B ; B)$ and $H C_{i}(B)$, respectively. The Hochschild homology dimension of an algebra is established in the next definition.

Definition 10 ([2], Definition 3.1). Let $B$ be an algebra. Let $d(A)=\inf \left\{n \in \mathbb{Z}_{0} \mid H_{i}(B ; B)=0\right.$ for $\left.i>n\right\}$. The value $d(B)$ is called the Hochschild dimension of $B$.

Proposition 11 ([2], Theorem 3.4). Let $B$ be an algebra with an increasing filtration $F B=\left\{F_{p} B \mid\right.$ $\left.F_{-1} B=0, p \in \mathbb{Z}\right\}$. Suppose that $d(G(B))=n<\infty$. Then the natural map $H C_{i}\left(F_{0} B\right) \rightarrow H C_{i}(B)$ is an isomorphism for all $i \geq n$.

The key result of this paper is Proposition 12 which establishes the cyclic homology of skew $P B W$ extensions.
Proposition 12. Let $R$ be a ring. If $A$ is a skew $P B W$ extension of $R$ and $d(G(A))<\infty$, then $H C_{i}(A) \cong$ $H C_{i}(R)$ for all $i \geq d(G(A))$.

Proof. The result follows from Proposition 5 and Proposition 11.

The following example will be of great importance in the Section 4.

Example 13. Let $\mathbb{k}$ be a field, $Q=\left(q_{i, j}\right)_{1 \leq i, j \leq v}$ a family of elements of $\mathbb{k} \backslash\{0\}$ verifying $q_{i, i}=1$ and $q_{i, j} q_{j, i}=1$ for all $i<j$, and $X=$ $\left\{x_{1}, \ldots, x_{v}\right\}$ a set of $v$ indeterminates. The multiparametric affine space is the $\mathbb{k}$-algebra $S_{Q}(X)$ generated by $x_{1}, \ldots, x_{v}$ and the relations $x_{j} x_{i}=q_{i, j} x_{i} x_{j}, 1 \leq i<j \leq v$. Note that $S_{Q}(X)=$ $\sigma(\mathbb{k})\left\langle x_{1}, \ldots, x_{v}\right\rangle$.

When all the coefficients $q_{i, j}$ for $i<j$ are equal to a constant $q$, the Hochschild dimension of $S_{Q}(X)$ is equal to $v$ if $q$ is a root of unity, and it is equal to 1 if $q$ is not a root of unity ([10], Remark 3.1.1). In the general case we have

(i) $H H_{n}\left(S_{Q}(X)\right)=0$ for all $n>v$;

(ii) If $H H_{n}\left(S_{Q}(X)\right) \neq 0$, then $H H_{m}\left(S_{Q}(X)\right) \neq 0$ for all $m \leq n$.

(iii) The Hochschild dimension of $S_{Q}(X)$ is the greatest $n$ such that there exist indexes $1 \leq p_{1}<$ $\cdots<p_{n} \leq v$ and a family of natural members $m_{p_{i}}, 1 \leq i \leq n$ verifying

$$
\prod_{i=1}^{n} q_{p_{r}, p_{i}}^{m_{p_{i}}}=1 \quad \forall r \text { with } 1 \leq r \leq n
$$

(see [10], Corollary 3.1.4 for more details).

Remark 6, Proposition 12 and Example 13 guarantee the following result

Proposition 14. Let $A$ be a skew $P B W$ extension of type derivation over a field $\mathbb{k}$. Then $H C_{i}(A) \cong$ $H C_{i}(\mathbb{k})$ for $i \geq d\left(S_{Q}(X)\right)$.

\section{Examples}

In this section we present examples of skew PBW extensions where Proposition 12 and Proposition 14 can be applied. The complete references of all examples can be found in [13]. 


\section{1. $P B W$ Extensions}

Any $P B W$ extension $A=\sigma(R)\left\langle x_{1}, \ldots, x_{n}\right\rangle$ is a bijective skew $P B W$ extension since in this case $\sigma_{i}=\mathrm{id}_{R}$ for every $1 \leq i \leq n$, and $c_{i, j}=1$ for every $1 \leq i, j \leq n$. Thus, for $P B W$ extensions we have $A=\operatorname{id}_{R}(R)\left\langle x_{1}, \ldots, x_{n}\right\rangle$. Some particular examples of $P B W$ extensions are the polynomial rings, skew polynomial rings of derivation type, universal enveloping algebras, and differential operator rings.

We recall that a filtered algebra $B$ is called quasicommutative if its associated graded algebra is commutative. This is the case for $P B W$ extensions above, which follows from Proposition 5. The Hochschild homology and the cyclic homology of this type of algebras was computed in [11].

\section{Example 15.}

1. ([15], p. 10, 59). For a commutative ring $k$, $H H_{0}(k)=k$ and $H H_{n}(k)=0, n>0$. With respect to the cyclic homology, $H C_{2 n}(k)=k$, $H C_{2 n+1}(k)=0, n \geq 0$.

2. Let $\mathscr{U}(\mathfrak{g})$ be the universal enveloping algebra of a Lie algebra $\mathfrak{g}$ of dimension $n$ over a field $\mathbb{k}$ of characteristic 0 . Since $G(\mathscr{U}(\mathfrak{g}))$ is isomorphic to a polynomial ring $\mathbb{k}\left[x_{1}, \ldots, x_{n}\right]$, and $d\left(\mathbb{k}\left[x_{1}, \ldots, x_{n}\right]\right)=n$, then $H C_{i}(\mathscr{U}(\mathfrak{g})) \cong$ $H C_{i}(\mathbb{k})$ for $i \geq n$.

3. For any Lie algebra $\mathfrak{g}$, Kassel [11] computed the Hochschild and cyclic homology groups of its enveloping algebra in terms of the canonical Lie-Poisson structure on the dual $\mathfrak{g}^{*}$. For the first Weyl algebra, Kassel proves that if $\mathbb{k}$ is a field of characteristic zero, then

$$
\begin{aligned}
& H_{i}\left(A_{1}(\mathbb{k})\right)=\left\{\begin{array}{ll}
\mathbb{k}, & i=2, \\
0, & \text { other case, }
\end{array}\right. \text { and } \\
& H C_{i}\left(A_{1}(\mathbb{k})\right)= \begin{cases}\mathbb{k}, & i \text { is even, } i \geq 2, \\
0, & \text { other case. }\end{cases}
\end{aligned}
$$

Since $A_{1}(\mathbb{k}) \cong \sigma(\mathbb{k}[x])\langle y\rangle$, we have $G\left(A_{1}(\mathbb{k})\right)=$ $\mathbb{k}[x, y]$ and $d\left(G\left(A_{1}(\mathbb{k})\right)\right)=2$. Hence, Proposition 12 implies $H C_{i}\left(A_{1}(\mathbb{k})\right)=H C_{i}(\mathbb{k}), i \geq 2$, which coincides with (4.1) and [15], p. 10, 59.

4. Loday [15], p. 94, showed that for a field $\mathbb{k}$ containing the field of rational numbers $\mathbb{Q}$, the Hochschild homology and the cyclic homology of the Weyl algebra $A_{n}(\mathbb{k})$, are given by

$$
\begin{gathered}
H_{i}\left(A_{n}(\mathbb{k})\right)=\left\{\begin{array}{ll}
\mathbb{k}, & i=2 n, \\
0, & \text { otherwise }
\end{array}\right. \text { and } \\
H C_{i}\left(A_{n}(\mathbb{k})\right)= \begin{cases}\mathbb{k}, & i=2 j, j \geq n, \\
0, & \text { otherwise, }\end{cases}
\end{gathered}
$$

respectively. Since $A_{n}(\mathbb{k}) \cong \sigma\left(\mathbb{k}\left[x_{1}, \ldots, x_{n}\right]\right)$ $\left\langle y_{1}, \ldots, y_{n}\right\rangle \quad$ ([13], Section 3.1), then $G\left(A_{n}(\mathbb{k})\right) \cong \mathbb{k}\left[x_{1}, \ldots, x_{n}, y_{1}, \ldots, y_{n}\right]$. In this way $d\left(G\left(A_{n}(\mathbb{k})\right)\right)=2 n$, and by Proposition 12 we obtain $H C_{i}\left(A_{n}(\mathbb{k})\right)=H C_{i}\left(\mathbb{k}\left[x_{1}, \ldots, x_{n}\right]\right), i \geq 2 n$, which coincides with (4.2).

5. With respect to universal enveloping algebras, Kassel [11] computed its Hochschild homology and cyclic homology. He obtain exact values for the groups $H_{i}(\mathscr{U}(\mathfrak{s l}(2, \mathbb{k}))), H C_{i}(\mathscr{U}(\mathfrak{s l}(2, \mathbb{k})))$, $H_{i}(\mathscr{U}(\mathfrak{s o}(4)))$, and $H C_{i}(\mathscr{U}(\mathfrak{s o}(4))), i \geq 0$.

\subsection{Ore Extensions of Bijective Type}

Any skew polynomial ring $R[x ; \sigma, \delta]$ of bijective type ( $\sigma$ bijective) is a bijective skew $P B W$ extension. In this case we have $R[x ; \sigma, \delta] \cong \sigma(R)\langle x\rangle$. If additionally $\delta=0$, then $R[x ; \sigma]$ is quasi-commutative. In a general way, let $R\left[x_{1} ; \sigma_{1}, \delta_{1}\right] \cdots\left[x_{n} ; \sigma_{n}, \delta_{n}\right]$ be an iterated skew polynomial ring of bijective type, i.e., the following conditions hold:

- for $1 \leq i \leq n, \sigma_{i}$ is bijective;

- for every $r \in R$ and $1 \leq i \leq n, \sigma_{i}(r), \delta_{i}(r) \in R$;

- for $i<j, \sigma_{j}\left(x_{i}\right)=c x_{i}+d$, with $c, d \in R$ and $c$ has a left inverse;

- for $i<j, \delta_{j}\left(x_{i}\right) \in R+R x_{1}+\cdots+R x_{n}$,

then, $R\left[x_{1} ; \sigma_{1}, \delta_{1}\right] \cdots\left[x_{n} ; \sigma_{n}, \delta_{n}\right]$ is a bijective skew $P B W$ extension. Under these conditions we have $R\left[x_{1} ; \sigma_{1}, \delta_{1}\right] \cdots\left[x_{n} ; \sigma_{n}, \delta_{n}\right] \cong \sigma(R)\left\langle x_{1}, \ldots, x_{n}\right\rangle([13]$, Section 3.2). Therefore, by Remark 6 we have $G\left(\sigma(R)\left\langle x_{1}, \ldots, x_{n}\right\rangle\right) \cong R\left[z_{1} ; \sigma_{z_{1}}\right] \cdots\left[z_{n} ; \sigma_{z_{n}}\right]$, where $\sigma_{j}(r)=c_{j, r}$ and $\sigma_{j}\left(z_{i}\right)=c_{i, j} z_{i}$ for $1 \leq i<j \leq n$. By Proposition 12 we obtain

$$
\begin{array}{r}
H C_{i}\left(R\left[x_{1} ; \sigma_{1}, \delta_{1}\right] \cdots\left[x_{n} ; \sigma_{n}, \delta_{n}\right]\right) \cong H C_{i}(R), \\
\text { for } i \geq d\left(G\left(\sigma(R)\left\langle x_{1}, \ldots, x_{n}\right\rangle\right)\right) .
\end{array}
$$

Example 16. Some remarkable examples of this kind of noncommutative rings are the following:

1. Quantum plane $\mathscr{O}_{q}\left(\mathbb{k}^{2}\right)$. Let $q \in \mathbb{k} \backslash\{0\}$. The quantized coordinate ring of $\mathbb{k}^{2}$ is a $\mathbb{k}$-algebra, 
denoted by $\mathscr{O}_{q}\left(\mathbb{k}^{2}\right)$, presented by two generators $x, y$ and the relation $x y=q y x$. We have $\mathscr{O}_{q}\left(\mathbb{k}^{2}\right) \cong \sigma(\mathbb{k})\langle x, y\rangle, G\left(\mathscr{O}_{q}\left(\mathbb{k}^{2}\right)\right) \cong \mathbb{k}[y]\left[x ; \sigma_{x}\right]$. If $q$ is a root of the unity, $H C_{i}\left(\mathscr{O}_{q}\left(\mathbb{k}^{2}\right)\right) \cong$ $H C_{i}(\mathbb{k})$ for $i \geq 2$, and if $q$ is not a root of the unity, $H C_{i}\left(\mathscr{O}_{q}\left(\mathbb{k}^{2}\right)\right) \cong H C_{i}(\mathbb{k})$ for $i \geq 1$.

2. The algebra of q-differential operators $D_{q, h}[x, y]$. Let $q, h \in \mathbb{k}, q \neq 0$; consider the $\operatorname{ring} \mathbb{k}[y]\left[x ; \sigma_{x}, \delta\right]$, where $\sigma_{x}(y):=q y, \delta(y):=h$. Then $x y=\sigma_{x}(y) x+\delta(y)=q y x+h$ whence $x y-q y x=h$, and hence $D_{q, h}[x, y] \cong \sigma(\mathbb{k})\langle x, y\rangle$. In this way $G\left(D_{q, h}[x, y]\right) \cong \mathbb{k}[y]\left[x ; \sigma_{x}\right]$. Again, if $q$ is a root of the unity, then $H C_{i}\left(D_{q, h}[x, y]\right) \cong$ $H C_{i}(\mathbb{k})$ when $i \geq 2$, and if $q$ is not a root of the unity, $H C_{i}\left(D_{q, h}[x, y]\right) \cong H C_{i}(\mathbb{k})$ when $i \geq 1$.

3. The mixed algebra $D_{h}$. It is defined by $D_{h}:=\mathbb{k}[t]\left[x ; \operatorname{id}_{\mathbb{k}[t]}, \frac{d}{d t}\right]\left[x_{h} ; \sigma_{h}\right]$, where $h \in \mathbb{k}$ and $\sigma_{h}(x):=x$. Then $D_{h} \cong \sigma(\mathbb{k})\left\langle t, x, x_{h}\right\rangle, G\left(D_{h}\right) \cong$ $\mathbb{k}[t]\left[x ; \mathrm{id}_{\mathbb{k}[t]}\right]\left[x_{h} ; \sigma_{h}\right]$. If $q$ is a root of the unity, $H C_{i}\left(D_{h}\right) \cong H C_{i}(\mathbb{k})$ for $i \geq 3$. In other case, $H C_{i}\left(D_{h}\right) \cong H C_{i}(\mathbb{k})$ for $i \geq 1$.

Remark 17. Guccione and Guccione in [10] computed under certain conditions the Hochschild homology and the cyclic homology of Ore extensions. For instance, they proved ([10], Corollary 2.5) that if $\mathbb{k}$ is a field, $B$ a $\mathbb{k}$-algebra and $E=B[t ; \alpha, \delta]$ an Ore extension satisfying the following conditions:

- As a $\mathbb{k}$-module, $B$ is a direct $\operatorname{sum} B=\bigoplus_{s \in \mathbb{N}^{v}} B_{S}$, with $B_{0}=\mathbb{k}$,

- There exist $q_{1}, \ldots, q_{v} \in \mathbb{k} \backslash\{0\}$ such that $\alpha^{-1}(a)=q_{1}^{m_{1}} \cdots q_{v}^{m_{v}} a$, provided that $a \in$ $B_{\left(m_{1}, \ldots, m_{v}\right)}$,

and if $q_{1}^{m_{1}} \cdots q_{v}^{m_{v}}=1$ implies $m_{1}=\cdots=m_{v}=0$, then the Hochschild homology of $E$ with coefficients in $E$ is given by

$$
\begin{aligned}
& H H_{0}(E)=H H_{0}(B) \oplus \bigoplus_{r \geq 1} \mathbb{k} t^{r}, \\
& H H_{1}(E)=H H_{1}(B) \oplus \bigoplus_{r \geq 0} \mathbb{k} t^{r} \otimes t, \\
& H H_{n}(E)=H H_{n}(B), n>1 .
\end{aligned}
$$

The cyclic homology of $E$ is given by

$$
\begin{aligned}
& H C_{0}(E)=H C_{0}(B) \oplus \bigoplus_{r \geq 1} \mathbb{k} t^{r}, \\
& H C_{n}(E)=H C_{n}(B) \oplus \bigoplus_{r \geq 1} \frac{\mathbb{k}}{\langle r\rangle}, n>0 .
\end{aligned}
$$

\subsection{Operator Algebras}

In this subsection we recall some important and well-known operator algebras. Some of these algebras are skew $P B W$ extensions of fields and hence we can apply the result established in (4.3).

1. Algebra of linear partial differential operators. The $n$th Weyl algebra $A_{n}(\mathbb{k})$ over $\mathbb{k}$ coincides with the $\mathbb{k}$-algebra of linear partial differential operators with polynomial coefficients $\mathbb{k}\left[t_{1}, \ldots, t_{n}\right]$. As we have seen, the generators of $A_{n}(\mathbb{k})$ satisfy the following relations $t_{i} t_{j}=t_{j} t_{i}, \partial_{i} \partial_{j}=\partial_{j} \partial_{i}$, for $1 \leq i<$ $j \leq n$, and $\partial_{j} t_{i}=t_{i} \partial_{j}+\delta_{i j}$, for $1 \leq i, j \leq n$, where $\delta_{i j}$ is the Kronecker symbol. Therefore $\sigma(\mathbb{k})\left\langle t_{1}, \ldots, t_{n} ; \partial_{1}, \ldots, \partial_{n}\right\rangle$, its associated graded ring is isomorphic to $\mathbb{k}\left[t_{1}, \ldots, t_{n} ; \partial_{1}, \ldots, \partial_{n}\right]$ and $H C_{i}\left(\sigma(\mathbb{k})\left\langle t_{1}, \ldots, t_{n} ; \partial_{1}, \ldots, \partial_{n}\right\rangle\right) \cong H C_{i}(\mathbb{k})$, for $i \geq 2 n$.

2. Algebra of linear partial q-differential operators. For a fixed $q \in \mathbb{k} \backslash\{0\}$, this is the $\mathbb{k}$ algebra $\mathbb{k}\left[t_{1}, \ldots, t_{n}\right]\left[D_{1}^{(q)}, \ldots, D_{m}^{(q)}\right], n \geq m$, subject to the relations:

$$
\begin{aligned}
t_{j} t_{i} & =t_{i} t_{j}, & 1 \leq i<j \leq n, \\
D_{i}^{(q)} t_{i} & =q t_{i} D_{i}^{(q)}+1, & 1 \leq i \leq m, \\
D_{j}^{(q)} t_{i} & =t_{i} D_{j}^{(q)}, & i \neq j, \\
D_{j}^{(q)} D_{i}^{(q)} & =D_{i}^{(q)} D_{j}^{(q)}, & 1 \leq i<j \leq m .
\end{aligned}
$$

If $n=m$, this operator algebra coincides with the additive analogue $A_{n}\left(q_{1}, \ldots, q_{n}\right)$ of the Weyl algebra $A_{n}(q)$ (Section 4.5, Example (a)). This algebra can be expressed as the skew $P B W$ extension $\sigma(\mathbb{k})\left\langle t_{1}, \ldots, t_{n} ; D_{1}^{(q)}, \ldots, D_{m}^{(q)}\right\rangle$, and hence $H C_{i}\left(\sigma(\mathbb{k})\left\langle t_{1}, \ldots, t_{n} ; D_{1}^{(q)}, \ldots, D_{m}^{(q)}\right\rangle\right) \cong$ $H C_{i}(\mathbb{k}), i \geq n+m$.

3. Operator differential rings. Let $R$ be an algebra over a commutative ring $k$ and let $\Delta=$ $\left\{\delta_{1}, \ldots, \delta_{n}\right\}$ be a set of commuting derivations of $R$. Let $T=R\left[\theta_{1}, \ldots, \theta_{n} ; \delta_{1}, \ldots, \delta_{n}\right]$ be the operator differential ring. The elements of $T$ can be written in a unique way as left $R$-linear combinations with the ordered monomials in $\theta_{1}, \ldots, \theta_{n}$. The product on $T$ is defined extending the product from $R$ subject to the relation $\theta_{i} r-r \theta_{i}=\delta_{i}(r), r \in R, i=1, \ldots, n$, and $\theta_{i} \theta_{j}-\theta_{j} \theta_{i}=0, i, j=1, \ldots, n$, and $T=$ $\sigma(R)\left\langle\theta_{1}, \ldots, \theta_{n}\right\rangle$. Then $H C_{i}(T)=H C_{i}(R)$ for $i \geq n$. 


\subsection{Diffusion Algebras}

Diffusion algebras arose in physics as a possible way to understand a large class of 1-dimensional stochastic process. A diffusion algebra $\mathscr{A}$ with parameters $a_{i j} \in \mathbb{C} \backslash\{0\}, 1 \leq i, j \leq n$ is a $\mathbb{C}$-algebra generated by indeterminates $x_{1}, \ldots, x_{n}$ subject to relations $a_{i j} x_{i} x_{j}-b_{i j} x_{j} x_{i}=r_{j} x_{i}-r_{i} x_{j}$, whenever $i<j, b_{i j}, r_{i} \in \mathbb{C}$ for all $i<j$. Therefore $\mathscr{A}$ admits a $P B W$-basis of standard monomials $x_{1}^{i_{1}} \cdots x_{n}^{i_{n}}$, that is, $\mathscr{A}$ is a diffusion algebra if these standard monomials are a $\mathbb{C}$-vector space basis for $\mathscr{A}$. From Definition 1, (iii) and (iv), it is clear that the family of skew $P B W$ extensions are more general than diffusion algebras.

In the applications to physics the parameters $a_{i j}$ are strictly positive reals and the parameters $b_{i j}$ are positive reals as they are unnormalised measures of probability. We will denote $q_{i j}:=\frac{b_{i j}}{a_{i j}}$. The parameter $q_{i j}$ is a root of unity if and only if is equal to 1. It is therefore reasonable that we will sometimes assume these parameters not to be a root of unity other than 1. If all coefficients $q_{i j}$ are nonzero, then the corresponding diffusion algebra have a $P B W$ basis of standard monomials $x_{1}^{i_{1}} \cdots x_{n}^{i_{n}}$ and hence these algebras are skew $P B W$ extensions, but not Ore extensions (see [13]). Therefore, $H C_{i}(\mathscr{A})=H C_{i}(\mathbb{C})$ for $i \geq d(G(\mathscr{A}))$.

\subsection{Quantum Algebras}

The term quantum group is usually used, not only for the $q$-analogue of the coordinate ring of a semisimple algebraic group but also for the $q$ analogue of the universal enveloping algebra of a semisimple Lie algebra. A quantum group is a Hopf algebra but in this article we will only concerned with the algebra structure and so the coalgebra structure will not be considered. We remark that the exact value of the Hochschild dimension of the associated graded ring of every skew $P B W$ extension $A$, that is, $d(G(A))$ can be found it using Example 13.

\section{Example 18.}

1. Additive analogue of the Weyl algebra. The $\mathbb{k}-$ algebra $A_{n}\left(q_{1}, \ldots, q_{n}\right)$ is generated by the variables $x_{1}, \ldots, x_{n}, y_{1}, \ldots, y_{n}$ subject to the relations:

$$
\begin{array}{lrl}
x_{j} x_{i}=x_{i} x_{j}, & 1 \leq i, j \leq n, \\
y_{j} y_{i}=y_{i} y_{j}, & 1 \leq i, j \leq n, \\
y_{i} x_{j}=x_{j} y_{i}, & i \neq j, \\
y_{i} x_{i}=q_{i} x_{i} y_{i}+1, & 1 \leq i \leq n,
\end{array}
$$

where $q_{i} \in \mathbb{k} \backslash\{0\}$. In this way we have $A_{n}\left(q_{1}, \ldots, q_{n}\right) \cong \sigma(\mathbb{k})\left\langle x_{1}, \ldots, x_{n}, y_{1}, \ldots, y_{n}\right\rangle$, that is, $A_{n}\left(q_{1}, \ldots, q_{n}\right)$ is a skew $P B W$ extension of the field $\mathbb{k}$.

2. Quantum algebra $\mathscr{U}^{\prime}(\mathfrak{s o}(3, \mathbb{k}))$. This algebra is the $q$-analogue of the universal enveloping algebra $\mathfrak{s o}(3, \mathbb{k})$. By definition it is the $\mathbb{k}$-algebra generated by the variables $I_{1}, I_{2}, I_{3}$ subject to relations $I_{2} I_{1}-q I_{1} I_{2}=-q^{1 / 2} I_{3}$, $I_{3} I_{1}-q^{-1} I_{1} I_{3}=q^{-1 / 2} I_{2}, I_{3} I_{2}-q I_{2} I_{3}=-q^{1 / 2} I_{1}$, where $q \in \mathbb{k} \backslash\{0\}$. We have $\mathscr{U}^{\prime}(\mathfrak{s o}(3, \mathbb{k})) \cong$ $\sigma(\mathbb{k})\left\langle I_{1}, I_{2}, I_{3}\right\rangle$.

3. Dispin algebra $\mathscr{U}(\operatorname{osp}(1,2))$. This algebra is generated by $x, y, z$ over the commutative ring $k$ satisfying the relations $y z-z y=z, z x+$ $x z=y, x y-y x=x$. Thus, $\mathscr{U}(\operatorname{osp}(1,2)) \cong$ $\sigma(\mathbb{k})\langle x, y, z\rangle$.

4. Woronowicz algebra $\mathscr{W}_{v}(\mathfrak{s l}(2, \mathbb{k}))$. This algebra is generated by $x, y, z$ subject to the relations $x z-v^{4} z x=\left(1+v^{2}\right) x, x y-v^{2} y x=v z$, $z y-v^{4} y z=\left(1+v^{2}\right) y$, where $v \in \mathbb{k}-\{0\}$ is not a root of unity. Under certain conditions on $v$ we have the isomorphism $\mathscr{W}_{v}(\mathfrak{s l}(2, \mathbb{k})) \cong$ $\sigma(\mathbb{k})\langle x, y, z\rangle$.

5. q-Heisenberg algebra. The $\mathbb{k}$-algebra $H_{n}(q)$ is generated by the set of variables $x_{1}, \ldots, x_{n}$, $y_{1}, \ldots, y_{n}, z_{1}, \ldots, z_{n}$ subject to the relations: $x_{j} x_{i}=x_{i} x_{j}, z_{j} z_{i}=z_{i} z_{j}, y_{j} y_{i}=y_{i} y_{j}, 1 \leq i, j \leq n$, $z_{j} y_{i}=y_{i} z_{j}, z_{j} x_{i}=x_{i} z_{j}, y_{j} x_{i}=x_{i} y_{j}, \quad i \neq j$, $z_{i} y_{i}=q y_{i} z_{i}, z_{i} x_{i}=q^{-1} x_{i} z_{i}+y_{i}, y_{i} x_{i}=q x_{i} y_{i}, 1 \leq$ $i \leq n$, with $q \in \mathbb{k} \backslash\{0\}$. Then $H_{n}(q) \cong$ $\sigma(\mathbb{k})\left\langle x_{1}, \ldots, x_{n} ; y_{1}, \ldots, y_{n} ; z_{1}, \ldots, z_{n}\right\rangle$.

\subsection{3-Dimensional Skew Polynomial Algebras}

The universal enveloping algebra $\mathscr{U}(\mathfrak{s l}(2, \mathbb{k}))$ of the Lie algebra $\mathfrak{s l}(2, \mathbb{k})$, the Woronowicz's algebra $\mathscr{W}_{v}(\mathfrak{s l}(2, \mathbb{k}))$ and the dispin algebra $\mathscr{U}(\operatorname{osp}(1,2))$, are examples of algebras classified by Smith and Bell which are known as 3-dimensional skew polynomial algebras. Next we recall the definition of this algebras and we will see that they are particular examples of skew $P B W$ extensions. 
Definition 19. A 3-dimensional skew polynomial algebra $\mathscr{A}$ is the $\mathbb{k}$-algebra generated by the variables $x, y, z$ restricted to relations

$$
y z-\alpha z y=\lambda, \quad z x-\beta x z=\mu, \quad x y-\gamma y x=v,
$$

such that

1. $\lambda, \mu, v \in \mathbb{k}+\mathbb{k} x+\mathbb{k} y+\mathbb{k} z$, and $\alpha, \beta, \gamma \in \mathbb{k}^{*}$;

2. Standard monomials $\left\{x^{i} y^{j} z^{l} \mid i, j, l \geq 0\right\}$ are a $\mathbb{k}$-basis of the algebra.

It is clear from Definition 4.4 that 3-dimensional skew polynomial ring are skew $P B W$ extensions of the field $\mathbb{k}$. Hence, $H C_{i}(\mathscr{A})=H C_{i}(\mathbb{k})$ for $i \geq$ $d(G(\mathscr{A}))$.

Finally, we recall Proposition 20 which establishes a classification of 3-dimensional skew polynomial algebras.

Proposition 20 ([21], Theorem C.4.3.1, p. 101). Let $\mathscr{A}$ be a 3-dimensional skew polynomial algebra. Then $\mathscr{A}$ is one of the following algebras:

(a) if $|\{\alpha, \beta, \gamma\}|=3$, then $\mathscr{A}$ is defined by

$$
y z-\alpha z y=0, \quad z x-\beta x z=0, \quad x y-\gamma y x=0 .
$$

(b) if $|\{\alpha, \beta, \gamma\}|=2 y \beta \neq \alpha=\gamma=1, \mathscr{A}$ is one of the following algebras:

(i) $y z-z y=z, \quad z x-\beta x z=y, \quad x y-y x=x$;

(ii) $y z-z y=z, \quad z x-\beta x z=b, \quad x y-y x=x$;

(iii) $y z-z y=0, \quad z x-\beta x z=y, \quad x y-y x=0$;

(iv) $y z-z y=0, \quad z x-\beta x z=b, \quad x y-y x=0$;

(v) $y z-z y=a z, \quad z x-\beta x z=0, \quad x y-y x=x$;

(vi) $y z-z y=z, \quad z x-\beta x z=0, \quad x y-y x=0$.

Here $a, b$ are any elements $\mathbb{k}$. All nonzero values of $b$ give isomorphic algebras.

(c) If $|\{\alpha, \beta, \gamma\}|=2$ and $\beta \neq \alpha=\gamma \neq 1$, then $\mathscr{A}$ is one of the following algebras:

(i) $y z-\alpha z y=0, \quad z x-\beta x z=y+b, \quad x y-$ $\alpha y x=0$

(ii) $y z-\alpha z y=0, \quad z x-\beta x z=b, \quad x y-\alpha y x=$ 0.

In this case $b$ is an arbitrary element of $\mathbb{k}$. Again, any nonzero values of $b$ given isomorphic algebras.

(d) If $\alpha=\beta=\gamma \neq 1$, then $\mathscr{A}$ is the algebra

$y z-\alpha z y=a_{1} x+b_{1}, \quad z x-\alpha x z=a_{2} y+b_{2}$, $x y-\alpha y x=a_{3} z+b_{3}$.
If $a_{i}=0, i=1,2,3$, all nonzero values of $b_{i}$ give isomorphic algebras.

(e) If $\alpha=\beta=\gamma=1, \mathscr{A}$ is isomorphic to one of the following algebras

(i) $y z-z y=x, \quad z x-x z=y, \quad x y-y x=z$;

(ii) $y z-z y=0, \quad z x-x z=0, \quad x y-y x=z$;

(iii) $y z-z y=0, \quad z x-x z=0, \quad x y-y x=b$;

(iv) $y z-z y=-y, \quad z x-x z=x+y, \quad x y-y x=0$;

(v) $y z-z y=a z, \quad z x-x z=z, \quad x y-y x=0$;

Parameters $a, b \in \mathbb{k}$ are arbitrary and all nonzero values of $b$ generates isomorphic algebras.

\section{References}

[1] V. A. Artamonov, "Derivations of skew PBW extensions", Communications in Mathematics and Statistics, vol. 3, pp. 449-457, 2015.

[2] J. Block, "Cyclic Homology of Filtered Algebras", K-Theory, vol. 1, pp. 515-518, 1987.

[3] A. Connes, "Cohomologie Cyclique et Functeurs de Ext" ", C. R. Math. Acad. Sci. Paris, vol. 296 , pp. 953-958, 1983.

[4] A. Connes, "Non-commutative Differential Geometry", Publ. Math. IHES, vol. 62 , pp. $257-$ 360, 1985.

[5] B.L. Feigin and B.L. Tsygan, "Additive KTheory, in K-Theory: Arithmetic and Geometry", Lecture Notes in Math, vol. 1289 , pp. 67-209, 1988

[6] P. Feng and B. Tsygan, "Hochschild and Cyclic Homology of Quantum Groups", Commun. Math. Phys., vol. 140, pp. 481-521, 1991.

[7] C. Gallego and O. Lezama, "Gröbner Bases for Ideals of $\sigma$-PBW Extensions", Comm. Algebra, vol. 39, no. 1, pp. 50-75, 2011.

[8] N. R. González, Y. P. Suárez, "Ideales en el Anillo de Polinomios Torcidos $R[x ; \sigma, \delta]$ ", Ciencia en Desarrollo, vol. 5, no. 1, pp. 3137, 2014.

[9] J.A. Guccione and J.J. Guccione, "Hochschild and Cyclic Homology of $\mathbb{Q}$-difference Operators", J. Math. Sci. Univ. Tokyo, vol. 35, no. 3, pp. 413-422, 1995.

[10] J.A. Guccione and J.J. Guccione, "Hochschild and Cyclic Homology of Ore Extensions and Some Examples of Quantum Algebras", $K$ Theory, vol. 12, pp. 259-276, 1997. 
[11] C. Kassel, "L'homologie Cyclique des Algèbres Enveloppantes", Invent. Math., vol. 91, pp. 221-251, 1988.

[12] O. Lezama, J.P. Acosta and A. Reyes, "Prime ideals of skew PBW extensions", Rev. Un. Mat. Argentina, vol. 56, no. 2, pp. 39-55, 2015.

[13] O. Lezama and A. Reyes, "Some Homological Properties of Skew PBW Extensions", Comm. Algebra, vol. 42, pp. 1200-1230, 2014.

[14] Loday, J.-L. and Quillen, D., "Homologie Cyclique et Homologie de L'Algèbre de Lie des Matrices", C. R. Math. Acad. Sci. Paris, vol. 296, pp. 295-297, 1983.

[15] J.-L. Loday, Cyclic Homology. SpringerVerlag, sec.edit., 1998.

[16] D. Quillen, "Higher Algebraic $K$-Theory, in Algebraic K-Theory", Lecture Notes in Math. vol. 341, Springer, Berlin 1973.

[17] A. Reyes, "Gelfand-Kirillov Dimension of Skew PBW Extensions", Rev. Col. Mat. vol. 47, no. 1, pp. 95-111, 2013.

[18] A. Reyes, "Uniform dimension over skew PBW extensions", Rev. Col. Mat., vol. 48, no. 1, pp. 79-96, 2014.
[19] A. Reyes, "Jacobson's conjecture and skew PBW extensions", Rev. Integr. Temas Mat, vol. 32, no. 2, 139-152, 2014.

[20] A. Reyes, "Skew PBW Extensions of Baer, quasi-Baer, p.p. and p.q.-rings", Rev. Integr. Temas Mat., vol. 33, no. 2, pp. 173-189, 2015.

[21] A. L. Rosenberg, "Non-commutative Algebraic Geometry and Representations of Quantized Algebras". Math. Appl., vol. 330. Kluwer Academic Publishers, 1995.

[22] H. Suárez, O. Lezama, A. Reyes, "Some relations between N-Koszul, Artin-Schelter regular and Calabi-Yau algebras with skew PBW extensions", Ciencia en Desarrollo, vol. 6, no. 2, pp. 205-213, 2015.

[23] B.L. Tsygan, "Homology of Matrix LieAlgebras over Rings and Hochschild Homology", Uspekhi Mat. Nauk, vol. 38, pp. 217-218, 1983.

[24] C. Venegas, "Automorphisms for Skew PBW Extensions and Skew Quantum Polynomial Rings", Comm. Algebra, vol. 43, no. 5, pp. 1877-1897, 2015. 